\title{
Locally advanced cancer of the tongue base: A new method of surgical treatment
}

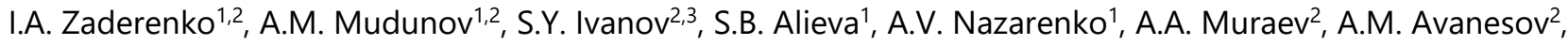 \\ V.T. Tsiklauri ${ }^{1}$, A.E. Kazimov ${ }^{1}$
}

\begin{abstract}
Introduction: Patients are characterized by locally advanced tumours in $70-80 \%$ of cases at presentation. Thus, the possibilities for curing and surgical treatment are limited. Total glossectomy, which involves tongue base resection, is associated with severe and permanent disabilities. Total glossectomy leads to severe dysphagia, alalia and social maladjustment. A number of issues motivated us to develop a new method of surgical treatment of locally advanced base of tongue cancer.

Objective: To introduce new opportunities for surgical treatment of locally advanced cancer of the tongue base.

Materials and Methods: Glossectomy was accomplished in five patients suffering from tongue cancer and admitted to N.N. Blokhin National Medical and Research Center of Oncology. Swallowing and speech were preserved in all 5 cases.

Results: We developed a new technique of surgical treatment of locally advanced cancer of the tongue base.

Conclusion: The developed surgical technique allows early rehabilitation. Patients are able to breath effortlessly, swallow and speak. Permanent tracheostomy and PEG tubes are not necessary. All patients remained socially active.
\end{abstract}

Keywords: Oropharyngeal neoplasms, tongue cancer, total glossectomy

\section{INTRODUCTION}

Oropharyngeal neoplasms are characterized by diverse histological types. Epithelial tumours, including squamous cell carcinomas of various differentiation statuses, are the most common $(90 \%)$ malignant neoplasms of the oral cavity. Sarcoma, melanoma, minor salivary gland carcinomas, and vascular and lymphogenic tumours are less common (1).

Patients are characterized by locally advanced tumours in $70-80 \%$ of cases at initial examination. Thus, cure and surgical treatment options are limited (2). Most patients receive conservative therapy, which exhibits limited efficacy (3). Isolated tongue base cancer is an uncommon disease. The presence of ecto-endodermal decussation in the area of the foramen cecum of the tongue prevents the application of non-surgical treatment (tumours located in this area respond poorly to radio- and chemotherapy).

Total glossectomy and tongue base resection are associated with severe and permanent disability. Such surgical procedures lead to severe dysphagia, alalia and social maladjustment. Patients frequently require gastrostomy, which also has a negative impact on quality of life $(4,5)$. Therefore, our aim was to enumerate poor outcomesto motivate the development of a new method of surgical treatment for locally advanced cancer of the tongue base.

\section{MATERIALS AND METHODS}

The development of a new method refers to medicine, especially oncology, plastic surgery, maxillo-facial surgery, stomatology, and rehabilitation medicine, and the newly developed method must be applicable to reconstructive and

\footnotetext{
1 Federal State Budgetary Institution «N.N. Blokhin National Medical and Research Center of Oncology» of the Ministry of Healthcare of the Russian Federation, Moscow, 115478, Kashirskoe shosse 23, Russia.

2 RUDN University, Department of Maxillo-facial Surgery and Operative Dentistry, Moscow, 117198, Miklukho-Maklaya Street 10, Russia

3 I.M. Sechenov First Moscow State Medical University, 8/2 Trubetskaya St., 119991 Moscow, Russia.
}

\author{
Correspondence: I.A. Zaderenko \\ Federal State Budgetary Institution «N.N. Blokhin National Medical and Research \\ Center of Oncology» of the Ministry of Healthcare of the Russian Federation, Moscow \\ 115478, Kashirskoe shosse 23, Russia. \\ RUDN University, Department of Maxillo-facial Surgery and Operative Dentistry,
} Moscow, 117198, Miklukho-Maklaya Street 10, Russia

Received: 11 Sep 2018, Accepted: 13 Nov 2018

(C) 2019 by the authors; licensee Modestum Ltd., UK. This article is an open access article distributed under the terms and conditions of the Creative Commons Attribution License (http://creativecommons.org/licenses/by/4.0/). 


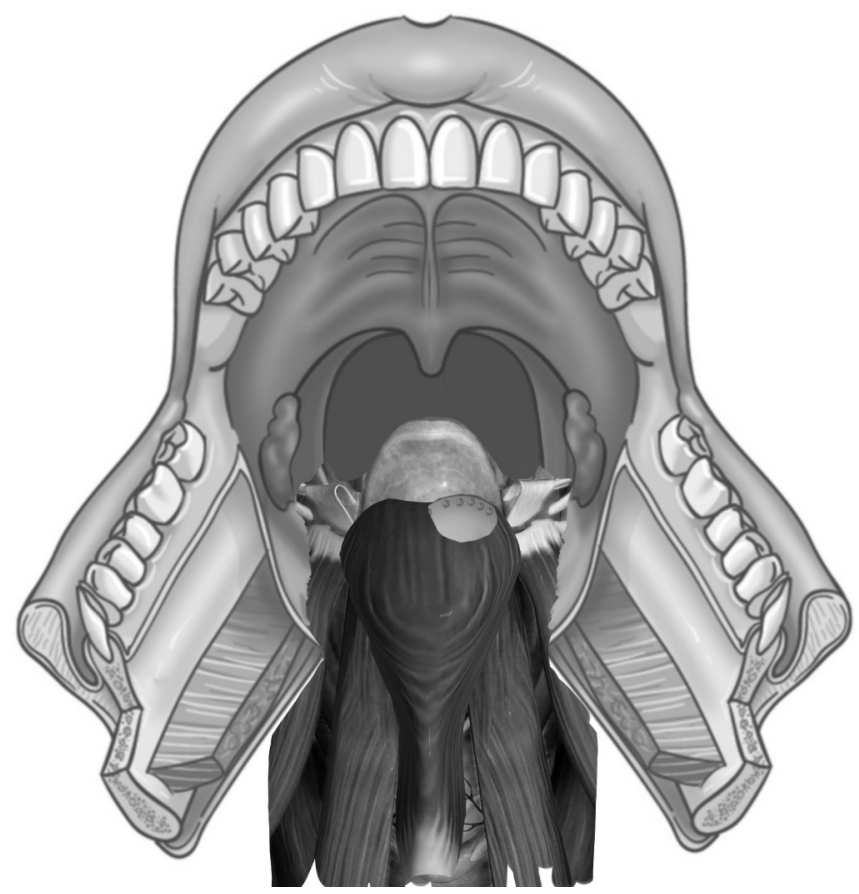

Figure 1: The resectional margins are $2 \mathrm{~cm}$ from tumor in accordance with principles of ablastics

restorative surgery. We seek to develop technology that offers improved treatment efficacy and functional rehabilitation of patients with locally advanced cancer of the base of the tongue. The developed surgical technique will stop reserve swallowing and speech, reduce surgical injury and avoid additional surgical interventions, such as flap remodelling and reinnervation. Furthermore, we observed a reduced duration of hospitalization, rehabilitation time and adjuvant radiotherapy interval, which are of prime importance in cases of malignant neoplasms.

This goal can be achieved by bilateral modified radical neck dissection that is indicated in case of median line involvement. The next step includes median line incision of the lower lip, mucous of the oral vestibule and skin of the mental region, skeletonization of the mental part of mandible and osteotomy between central incisors of mandible. This surgical approach is highly ablastic and permits adequate implementation of the proposed surgical technique. Next, the mandible fragments are forced apart, and the tongue is pull out of the oral cavity. The tongue position is fixed, and complete glossectomy is performed by cutting the tongue from the epiglottis and floor of the mouth. The resectional margin should exceed $2 \mathrm{~cm}$ in accordance with principles of ablastics (Figure 1). Then, the soft tissues located around the defect and form the floor of the mouth must be mobilized.

Epiglottis displacement due to the movement of the tongue is essential for swallowing and speech. In cases of total glossectomy for locally advanced tongue base cancer, the surgeon needs to cut the tongue from the epiglottis. This practice leads to the loss of the separatory function of the epiglottis.

However, preserved fragments of contralateral skeletal muscles (i.e., muscles that begin from bones, entwine the body of tongue and capable of changing the tongue position, including $\mathrm{m}$. Hyoglossus, $\mathrm{m}$. Styloglossus, m. Stylohyoideus and $\mathrm{m}$. Constrictor pharyngis superior) are separated from adjacent tissues and mobilized (Figure 2). 


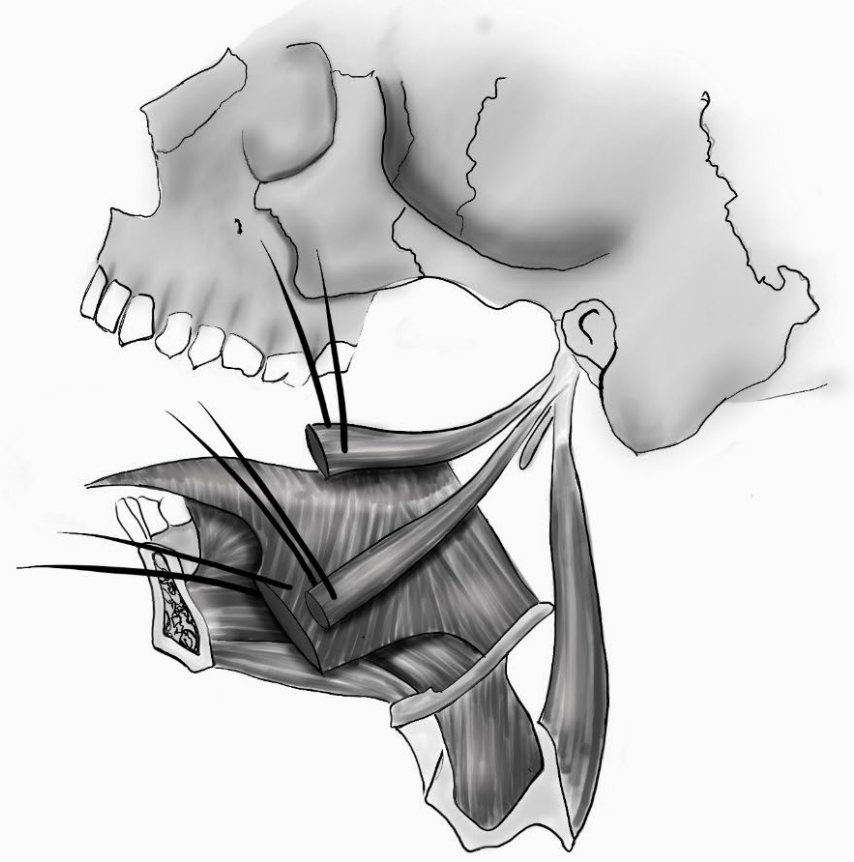

Figure 2: The muscles m. Hyoglossus, $m$. Styloglossus, $m$. Stylohyoideus and $m$. Constrictor pharyngis superior are separated from adjacent tissues and mobilized

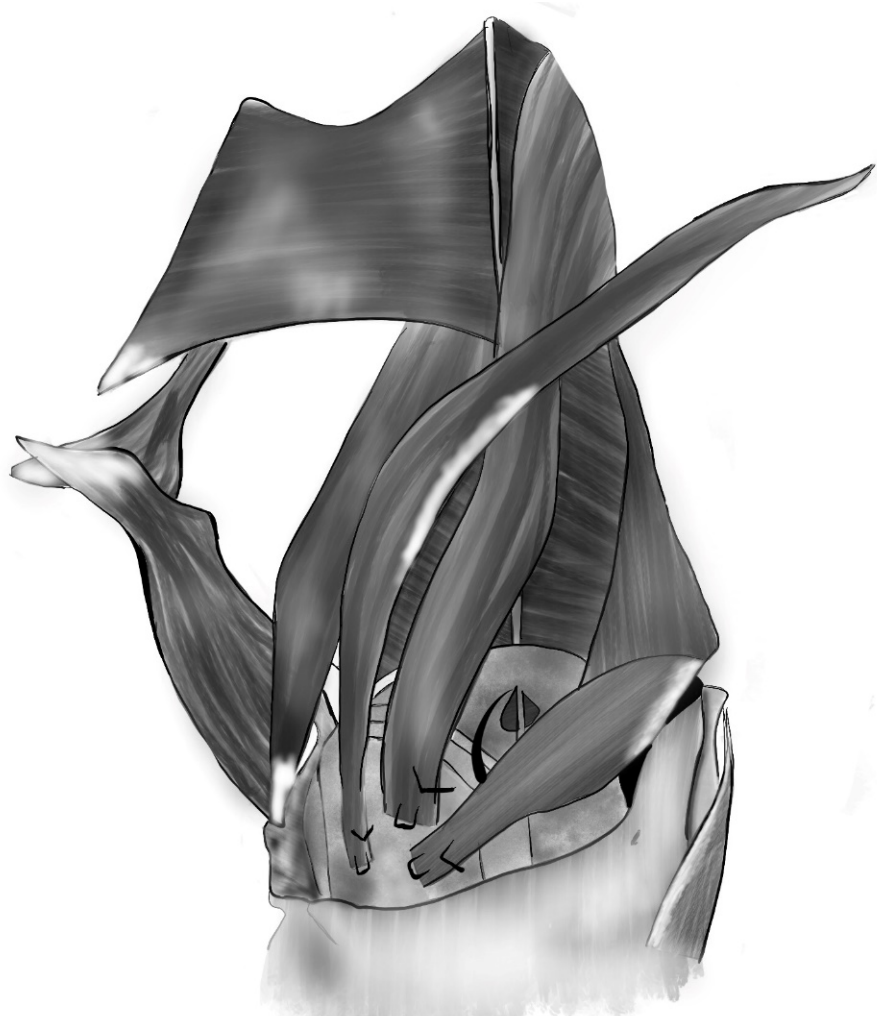

Figure 3: The fixation of mobilized muscle fragments to residual portions of epiglottic muscles (m. Arytenoideus obliguus and $m$. Thyroepiglotticus) and epiglottic cartilage with separate $U$ shaped stitches. Stitches are located on semicircle of the epiglottis at «4-5-6-7 hours»

The main stage of the operation includes the fixation of mobilized muscle fragments to residual portions of epiglottic muscles ( $\mathrm{m}$. Arytenoideusobliguus and $\mathrm{m}$. Thyroepiglotticus) and epiglottic cartilage with separate U-shaped stitches. Stitches must be evenly located on semicircle of the epiglottis at «4-5-6-7 hours» (Figure 3). It is crucial that surgeon's needle should not stick the posterior surface of the epiglottis. 


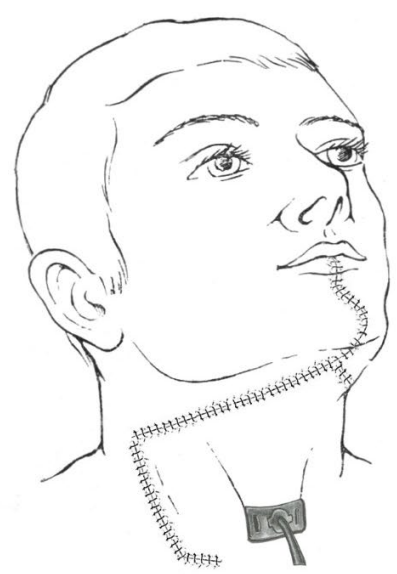

Figure 4: The concluding stage of operation includes stitching of the mouth floor, osteosynthesis of jawbone fragments and neck wound closing. As the operation involves the tongue base, it is necessary to perform tracheostomy to prevent asphyxia

The concluding stage of operation includes stitching of the mouth floor, osteosynthesis of jawbone fragments and neck wound closing. As the operation involves the tongue base, it is necessary to perform tracheostomy to prevent asphyxia (Figure 4).

\section{RESULTS}

According to this technique, 8 patients were operated from them - 3 women (37.5\%) and $5(62.5 \%)$ men. One male is 30 years of age, the rest are over 60 years old. For all patients, the tracheostomic tube was removed within 10-14 days. Swallowing in all patients is restored in up to 21 days. Within 2 years all patients are alive without relapse and metastases.

In the postoperative period, the following disturbances of sound reproduction were noted (in the place where consonant sounds are formed):

labial-labial: b, b', n, n', m, m '(preserved);

labial-dental: $v, v^{\prime}, f, f^{\prime}$ (are kept);

lingual-dental: $d, d^{\prime}, t, t^{\prime}$ (substitutions on $\left.b, b^{\prime}, p, p^{\prime}\right)$;

linguistically-dental: $z, z^{\prime}, c, c^{\prime}$ (are distorted);

linguistically alveolar: $r, r^{\prime}, l, l^{\prime}$ (are absent);

lingual-forehead: $z h$, sh, shch, ch (are distorted);

linguistically mediocre: $g^{\prime}$ (replacement of $\left.b^{\prime}\right), k^{\prime}\left(\right.$ replacement to $\left.p^{\prime}\right), h$ (preserved);

linguistically backward: $g$ (substitution of $b$ ), $k$ (replacement of $p$ ), $x$ (preserved).

All the explosive linguistic consonants $k^{\prime} k^{\prime}, g, g^{\prime}, t, t^{\prime}, d, d^{\prime}$ are replaced by the labial-labial consonants $p, p^{\prime}, b, b^{\prime}$ respectively. In this case, patients remain differentiated on the basis of deafness and voicelessness. Nasal lingual-dental consonants $n, n^{\prime}$, are replaced by nasal labia-labial $m, m^{\prime}$. As a result of formed replacements, improvement of syllable intelligibility was noted. Patients are advised to speak slowly, in short sentences. In this case, based on the context and situation, the surrounding people better understand the meaning of the statement.

Logopedic status was polymorphic mechanical dyslalia.

The main advantage of the proposed technique is attributable to its functional activity. Specifically, the exposed muscle flap is innervated by different cranial nerves that are initially involved in swallowing. The functional activity of the swallowing is achieved by contraction of preserved parts of muscles that typically participate in swallowing (skeletal muscles of the tongue and pharyngeal constrictor muscles). These muscles are innervated by cranial nerves that are involved in swallowing, including the glossopharyngeal nerve (IX pair), vagus nerve (X pair), and hypoglossal nerve (XII pair) (6). Thus, reconstitution of the process of swallowing involves not only the mechanical movement but is also under control of the central nervous system.

Low surgery injury is the consequence of the application of soft tissues adjacent to post resectional defect for preservation of swallowing and speech. Tumour excision and dissection of the muscle flap is limited by one anatomic area, so there is no need to dissect tissue flaps from distant anatomic areas. Additional surgical injury and incisions are also absent. The above-mentioned aspects offer the possibility to reduce the surgical intervention time significantly. 


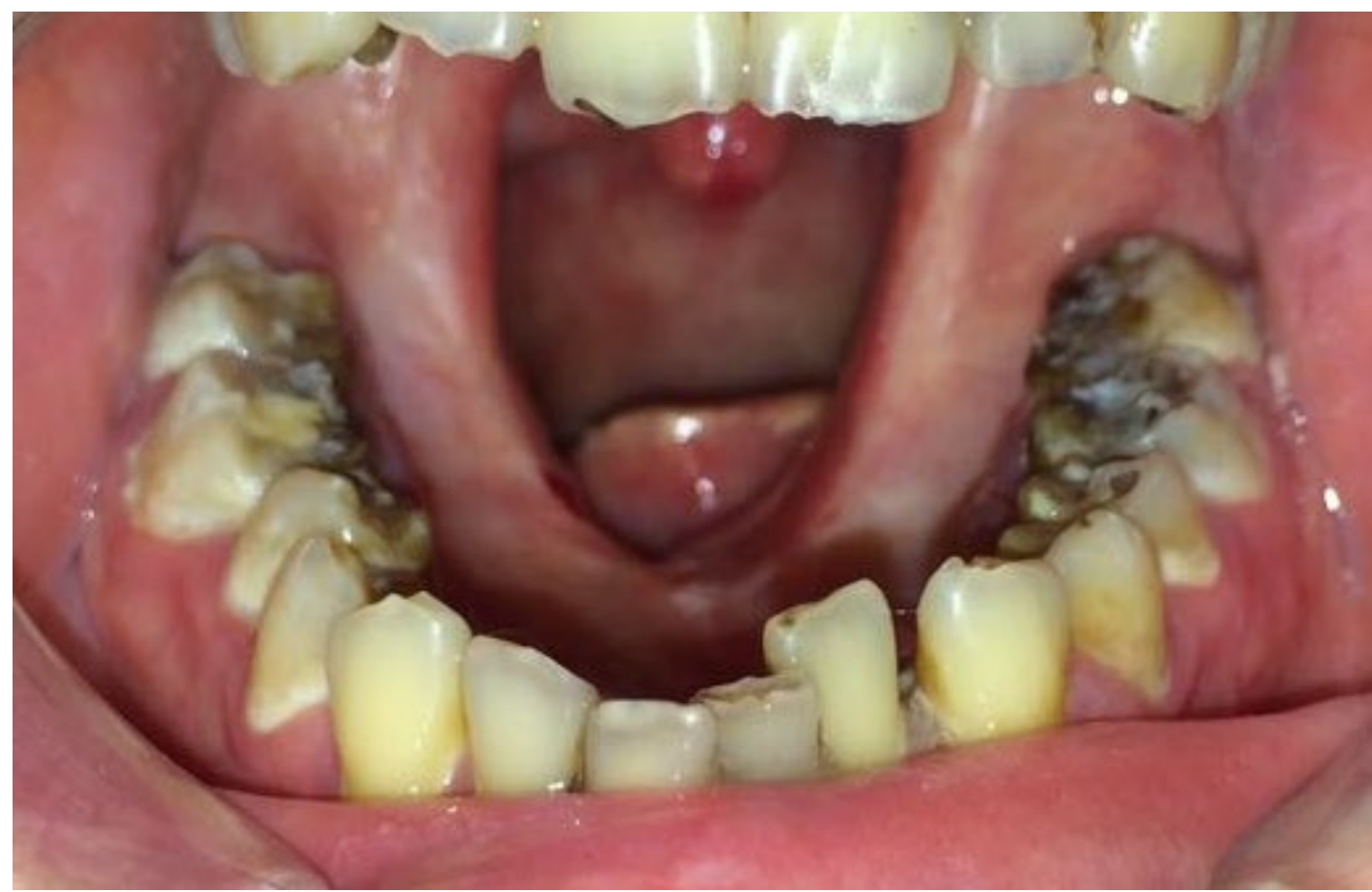

Figure 5: Intraoral view 1 mounth after operation

The proposed muscle flap is supplied by different arteries. Therefore, the risk of complications is low, and the healing process takes less time compared with the prototype. As a result, the hospitalization period can be reduced on average to 7-9 days.

Early repair allows the initiation of adjuvant radiotherapy within the time periods specified in treatment guidelines for locally advanced oropharyngeal cancer (i.e., 4-6 weeks after surgical treatment).

Swallowing and voice rehabilitation can be started in the immediate postoperative period after the removal of sutures (i.e., 9-11 days after surgery because this period is necessary for wound repair). Early functional load leads to fast rehabilitation (Figure 5).

\section{CONCLUSION}

The developed surgical technique for locally advanced carcinoma of the tongue base permits early rehabilitation. Patients are able to breath effortlessly, swallow and speak. Permanent tracheostomy and PEG tubes are not needed. All patients remain socially active.

\section{REFERENCES}

1. Barnes L, Eveson JW, Reichart P, Sidransky D. World Health Organization. Classification of Tumors. Pathology and Genetics of Head and Neck Tumors. IARC Press: Lyon 2005.

2. Пачес А.И. Опухоли головы и шеи. Клиническое руководство. Издательство: Практическая медицина. 2013.

3. Adelstein DJ. Squamous cell head and neck cancer. 2005:358. Humana Press: Totowa, NJ. https://doi.org/10.1007/978-1-59259-938-7 PMCid:PMC1059448

4. Cristina LB, et al. Speech Intelligibility After Glossectomy and Speech Rehabilitation.-Arch Otolaryngol Head Neck Surg. 2001;127(7):877-83.

5. Derrick T, Lin et al. Long-term Functional Outcomes of Total Glossectomy With or Without Total Laryngectomy.JAMA Otolaryngol Head Neck Surg. 2015;141(9):797-803. https://doi.org/10.1001/jamaoto.2015.1463 PMid:26291031

6. Netter F. Atlas of Human Anatomy, 6th Edition. Saunders. 2014:640.

$\diamond \diamond \diamond \diamond \diamond \diamond \diamond$

http://www.ejgm.co.uk 